\title{
The Effects of Strontium Ranelate in Asian Women with Postmenopausal Osteoporosis
}

J. S. Hwang $\cdot$ J. F. Chen $\cdot$ T. S. Yang $\cdot$

D. J. Wu - K. S. Tsai - C. Ho $~$ C. H. Wu

S. L. Su C. J. Wang $\cdot$ S. T. Tu

Published online: 11 March 2009

(C) Springer Science+Business Media, LLC 2009

\section{Erratum to: Calcif Tissue Int (2008) 83(5):308-314 DOI 10.1007/s00223-008-9180-z}

In the October 2008 issue (volume 83/number 5, pp. 308314), an error occurred in Table 1. In line 12, Serum BSAP

The online version of the original article can be found under doi:10.1007/s00223-008-9180-z.

J. S. Hwang · J. F. Chen · C. Ho

Division of Endocrinology and Metabolism, Department of Internal Medicine, Chang Gung Memorial Hospital, Chang Gung University, Linkou, Chlayi, Kaohsiung,

Taiwan, ROC

e-mail: hwangjs@cgmh.org.tw

\section{T. S. Yang}

Department of Obstetrics and Gynecology,

Taipei Veterans General Hospital, School of Medicine,

National Yang-Ming University, Taipei, Taiwan, ROC

D. J. Wu $\cdot$ C. H. Wu

Departments of Internal Medicine and Family Medicine, National Cheng Kung University, Medical Center, Tainan, Taiwan, ROC
(ng/mL), the data should read " $15.4 \pm 0.07$ " (not 1.54) for Strontium ranelate and "18.8 $\pm 0.10 "$ (not 1.88) for Placebo.

We regret this error.

\section{K. S. Tsai}

Department of Internal Medicine, National Taiwan University Hospital, College of Medicine, National Taiwan University,

Taipei, Taiwan, ROC

S. L. Su · S. T. Tu ( $₫)$

Division of Endocrinology and Metabolism,

Department of Internal Medicine, Changhua Christian Hospital, 135 Nanhsiao Street, Changhua, Taiwan, Taiwan, ROC

e-mail: 10836@cch.org.tw

C. J. Wang

Department of Radiology, Chang Gung Memorial Hospital, Linkou, Chang Gung University, Taipei, Taiwan, ROC 